\title{
Rechtsprechung des Europäischen Gerichtshofes
}

\section{Urteil „Meeresverschmutzung durch Schiffe“ - weitere Klärung der Reichweite der strafrecht- lichen Kompetenz des Gemeinschaftsrechts}

\section{Wolfgang Bogensberger}

Dieses Urteil knüpft an das - aufsehenerregende - Urteil des Europäischen Gerichtshofs vom 13. September 2005 (C176/03) zum „Umweltschutz durch Strafrecht“ an. Darin hatte der Gerichtshof zwar darauf hingewiesen, dass das Strafrecht ebenso wie das Strafprozessrecht grundsätzlich nicht in die Zuständigkeit der Gemeinschaft fällt; der Gemeinschaftsgesetzgeber kann aber, wenn die Anwendung wirksamer, verhältnismäßiger und abschreckender strafrechtlicher Sanktionen durch die zuständigen nationalen Behörden eine zur Bekämpfung schwerer Beeinträchtigungen der Umwelt unerlässliche Maßnahme darstellt, die Mitgliedstaaten gleichwohl zur Einführung derartiger Sanktionen verpflichten, um die volle Wirksamkeit der von ihm in diesem Bereich erlassenen Rechtsnormen zu gewährleisten ${ }^{1}$.

Trotz dieses Grundsatz-Urteils blieben unterschiedliche Auffassungen zu Fragen der Reichweite der gemeinschaftsrechtlichen Zuständigkeit für strafrechtliche Maßnahmen bestehen. Diese Unklarheiten bezogen sich auf

- die „horizontale“ Reichweite: Besteht eine solche strafrechtliche Annex-Zuständigkeit der EG auch für andere gemeinschaftsrechtliche Politikbereiche oder ist sie auf den Umweltschutz beschränkt?

- die „vertikale“ Reichweite: Ist der Gemeinschaftsgesetzgeber nur ermächtigt, die Elemente des strafrechtlichen Tatbestandes vorzugeben oder kann er auch, wenn notwendig, die Art der Strafe (Geldstrafe, Freiheitsstrafe, sonstige Strafen) oder das Maß der in den nationalen Gesetzbüchern vorzusehenden Strafe (Strafrahmen) festlegen?

Um diese beiden für die Rechtsetzungspraxis überaus bedeutsamen Fragen zu klären, erhob die Kommission Ende 2005 eine Klage auf Nichtigerklärung des Rahmenbeschlusses

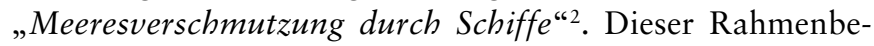
schluss verpflichtet die Mitgliedstaaten nicht nur, wirksame, angemessene und abschreckende strafrechtliche Sanktionen für natürliche und juristische Personen bei Meeresverschmutzung durch Schiffe vorzusehen; er legt zudem die Art und das $M a \beta$ der anzuwendenden strafrechtlichen Sanktionen fest, abgestuft nach Schwere der verursachten Schädigung von Menschen, Tier- und Pflanzenarten sowie der Wasserqualität.

Der Europäische Gerichtshof erklärte mit seinem Urteil vom 23. Oktober 2007 (C-440/05) - dem Grundgedanken des Urteils „Umweltschutz durch Strafrecht“ folgend - den Rahmenbeschluss „Meeresverschmutzung durch Schiffe“ für nichtig, weil dieser außerhalb des gemeinschaftsrechtlichen Rahmens erlassen wurde. Das Urteil weist darauf hin, dass die Verkehrspolitik zu den Grundlagen der Gemeinschaft gehört und der Gemeinschaftsgesetzgeber im Rahmen der ihm durch den EG-
Vertrag übertragenen Zuständigkeiten Maßnahmen zur Verbesserung der Sicherheit des Seeverkehrs ergreifen kann. Die Vorschriften des Rahmenbeschlusses, welche die Mitgliedstaaten verpflichten, bestimmte Verhaltensweisen strafrechtlich zu ahnden, hätten wirksam auf der Grundlage des EG-Vertrags erlassen werden können. Damit verstößt der Dritte-Säule Rahmenbeschluss gegen den Vorrang des Gemeinschaftsrechts. Allerdings fallen nach den - ohne jede nähere Begründung getroffenen - Ausführungen des Urteils die Bestimmung von Art und Maß der anzuwendenden strafrechtlichen Sanktionen nicht in die Zuständigkeit der Gemeinschaft. Insoweit verstößt der Rahmenbeschluss daher nicht gegen den Vorrang des Gemeinschaftsrechts; gleichwohl erklärte der Gerichtshof aber den gesamten Rahmenbeschluss wegen seiner Unteilbarkeit für nichtig.

Daraus ergibt sich zu den beiden strittigen Punkten folgende Klärung:

- Das Gemeinschaftsrecht hat eine strafrechtliche Zuständigkeit - neben dem Umweltschutz - auch in anderen gemeinschaftsrechtliche Politikbereichen, wenn diese zu den Grundlagen der Gemeinschaft zählen (wie im vorliegenden Fall die Verkehrspolitik) und soweit strafrechtliche Maßnahmen für deren effektive Durchführung notwendig sind;

- Das Gemeinschaftsrecht kann nur den strafrechtlichen Tatbestand vorgeben, nicht jedoch auch die Art oder das Maß der in den nationalen Gesetzbüchern vorzusehenden Strafe. Die europaweite Rechtsannäherung dazu bleibt, soweit dies erforderlich ist, einem Rechtsakt der Dritten Säule vorbehalten.

Die Grenzen des transnationalen ne-bis-in-idem Grundsatzes nach Artikel 54 des Schengener Durchführungsübereinkommens (SDÜ)

\section{Urteil Kraaijenbrink (C-367/05): Einheitlicher Vorsatz}

Frau Kraaijenbrink wurde 1998 in den Niederlanden wegen Drogenhandels und Geldwäsche zu einer Freiheitsstrafe von sechs Monaten verurteilt, deren Vollstreckung zur Bewährung ausgesetzt wurde. Drei Jahre später verurteilte sie ein belgisches Gericht wegen Geldwäsche zu einer Freiheitsstrafe von zwei Jahren, weil sie in Belgien Geldbeträge gewaschen hat, die aus dem Drogenhandel in den Niederlanden stammten.

Der belgische Kassationsgerichtshof stand vor der - das „idem“ betreffenden - Frage, ob die niederländische Verurteilung wegen Geldwäsche einem belgischen Strafverfahren entgegenstehe, auch wenn es sich in den Niederlanden und in Belgien offenbar weitgehend um verschiedene Taten (unterschiedliche Geldwäschehandlungen) handle, die jedoch die aufeinander folgende und fortgesetzte Durchführung ein und desselben Vorsatzes zu einer Straftat sind. Nach belgischem Strafrecht würde die Verbindung mehrerer Taten durch einen einheitlichen Vorsatz strafrechtlich eine Tat ergeben, aber gilt dies auch für Artikel 54 SDÜ? Sind verschiedene Taten insoweit „dieselbe Tat"?

Der Europäische Gerichtshof antwortete darauf mit Urteil vom 18. Juli 2007, dass ein einheitlicher Vorsatz noch nicht zwingend eine objektive Verbindung zwischen den Taten herstellt. Verschiedene Taten sind somit nicht schon deshalb „dieselbe Tat“, weil sie durch einen „einheitlichen Vorsatz“ verbunden sind. Eine Verurteilung auch in Belgien würde somit nicht gegen den transnationalen ne-bisin-idem Grundsatz des Artikels 54 SDÜ verstoßen. 


\section{Urteil Kretzinger (C-288/05): Vollstreckungs- voraussetzung ${ }^{3}$}

Herr Kretzinger war zweimal in Italien wegen Zigarettenschmuggels in Haft genommen und kurz darauf wieder auf freien Fuß gesetzt worden; danach wurde er in Italien in Abwesenheit zweimal zu Freiheitsstrafen verurteilt, einmal zu einer Freiheitsstrafe von einem Jahr und acht Monaten, deren Vollstreckung zur Bewährung ausgesetzt wurde, und das zweite Mal zu einer Freiheitsstrafe von zwei Jahren ohne Strafaussetzung zur Bewährung. Da Italien keinen Versuch unternommen hat, diese Freiheitsstrafe zu vollstrecken, ersuchte der vorlegende deutsche Bundesgerichtshof den Europäischen Gerichtshof um Klärung, welche Bedeutung die Vollstreckungsvoraussetzung des Artikels 54 SDÜ bei Freiheitsstrafen mit Bewährung hat und ob eine auf die Freiheitsstrafe ohne Bewährung anzurechnende kurzfristige Polizei- und Untersuchungshaft bereits die Sperrwirkung des Artikels 54 SDÜ auslöst bzw. welchen Einfluss der fortschreitende Ausbau der wechselseitigen Vollstreckungshilfe (etwa durch die Einführung des Europäischen Haftbefehls) auf die Vollstreckungsvoraussetzung hat.

Der Europäische Gerichtshof legte in seinem Urteil vom 18. Juli 2007 dazu fest, dass Freiheitsstrafen, deren Vollstreckung zur Bewährung ausgesetzt wurde, die Vollstreckungsvoraussetzung des Artikels 54 SDÜ erfüllen, weil sie „gerade vollstreckt" werden; sie lösen daher eine schengenweite Sperrwirkung aus. Hingegen wird diese Voraussetzung bei einer (noch nicht vollstreckten) Freiheitsstrafe ohne Strafaussetzung zur Bewährung nicht erfüllt, wenn der Angeklagte nur kurzfristig in Polizei- und/oder Untersuchungshaft genommen worden ist (sie wird weder "gerade vollstreckt" noch ist sie „bereits vollstreckt“). Ebenso wenig ist die Vollstreckungsvoraussetzung dadurch erfüllt, dass ein Mitgliedstaat, in dem eine Person verurteilt worden ist, die Möglichkeit hat, einen Europäischen Haftbefehl auszustellen, um diese Person zur Vollstreckung des Urteils festnehmen zu lassen.

(Diese Urteile sind alle abrufbar unter: http://curia.europa.eu/ jurisp/cgi-bin/form.pl?lang=de)

* Dr. Wolfgang Bogensberger ist Leiter der Straflegislativsektion im Bundesministerium für Justiz in Wien.

\section{Fußnoten}

1 Siehe dazu das EU-update in NKP 2/2007, S. 47 sowie Satzger in NKP 4/2007, S. 95

2 Rahmenbeschluss 2005/667/JI des Rates vom 12. Juli 2005 zur Verstärkung des strafrechtlichen Rahmens zur Bekämpfung der Verschmutzung durch Schiffe (ABl. L 255, 30.9.2005, S. 164).

3 Die Vollstreckungsvoraussetzung in Artikel 54 SDÜ lautet: „... vorausgesetzt, dass im Falle einer Verurteilung die Sanktion bereits vollstreckt worden ist, gerade vollstreckt wird oder nach dem Recht des Urteilsstaat nicht mehr vollstreckt werden kann."

\section{EU: Daten zur Kriminalität und Strafverfolgung 1995-2005}

EUROSTAT - das Statistische Amt der EU - veröffentlichte für den Zeitraum 1995 bis 2005 die Ergebnisse einer Datenerhebung zur Kriminalität und Strafverfolgung in der EU (genauer: in jenen Mitgliedstaaten der EU, die für diesen Zeitraum über relativ konsistente Daten verfügen). Dabei werden polizeilich registrierte Straftaten (Gesamtkriminalität, Tötungsdelikte, Gewaltdelikte, Raubdelikte, Wohungseinbrüche, Kraftfahrzeugdiebstahl und Drogenhandel) miteinander verglichen, ebenso wie die Gefangenenpopulation.

Die wichtigsten Ergebnisse dieses ersten Versuches eines Gesamtbildes für die EU:

- Die Entwicklung der Gesamtkriminalität im Zeitraum 1995 - 2005 deutet auf einen generellen Anstieg um rund ein halbes Prozent pro Jahr hin. In den meisten Ländern erreichte die Gesamtkriminalität um das Jahr 2002 einen Höhepunkt und ist seither leicht zurückgegangen. Am stärksten erhöhte sich die Gesamtkriminalität in Slowenien, Polen, Malta, Griechenland und Portugal, während sie in Ungarn, Dänemark und der Tschechischen Republik rückläufig war.

- Den Polizeiunterlagen zufolge betrug die jährliche Häufigkeit von Tötungsdelikten in den Jahren 2003 - 2005 rund 1,8 je 100000 Einwohner, war in den Hauptstädten allerdings höher (im Durchschnitt 2,2). Die höchsten Werte liegen in Litauen und Estland, die niedrigsten in Luxemburg, Österreich, Malta, Deutschland und Schweden.

- Zugenommen haben die registrierten Gewaltdelikte (Straftaten gegen die Person, Raub- und Sexualdelikte). Die Zahl dieser Straftaten erhöhte sich zwischen 1995 und 2005 jeweils um $4 \%$ bis $5 \%$. Die Raubdelikte nahmen stark in Polen, in der Slowakei, in Frankreich sowie in Portugal und Schweden zu, während sie in Rumänien und Deutschland zurückgingen.

- Dagegen nahmen die Eigentumsdelikte ab. So ging etwa die Zahl der Wohnungseinbrüche um durchschnittlich 3\% pro Jahr zurück, am Stärksten in Rumänien, Bulgarien und Belgien. Kraftfahrzeugdiebstähle gingen zwischen 1995 und 2005 noch stärker zurück, nämlich um 5\% pro Jahr; hier sind die stärksten Abnahmen in Bulgarien, Belgien sowie den Niederlanden und Deutschland zu beobachten.

- Der Drogenhandel hingegen hat zwischen 1995 und 2005 um rund 4\% pro Jahr zugenommen, am Stärksten in Bulgarien, Ungarn, Polen, Rumänien, der Tschechischen Republik und in Lettland.

- Die Gefangenenpopulation ist zwischen 1995 und 2005 beständig gewachsen, so dass in den Jahren 2003 - 2005 in den EU-Mitgliedstaaten auf 100000 Einwohner im Durchschnitt 124 Strafgefangene kamen. Die höchsten Werte finden sich in den drei baltischen Ländern, in Polen, in Rumänien, in der Tschechischen Republik und in der Slowakei sowie in Ungarn; unterdurchschnittlich oft werden Menschen in Slowenien, Zypern, Finnland, Dänemark, Malta, Nordirland und Irland sowie in Schweden gefangen genommen.

(Die unter der Rubrik „Bevölkerung und soziale Bedingungen" Nummer 15/2007 abgedruckte Statistik Kriminalität und Strafverfolgung ist abrufbar unter http://epp.eurostat. ec.europa.eu/cache/ITY_OFFPUB/KS-SF-07-015/DE/KS-SF07-015-DE.PDF). 